\title{
Implementation of Fuzzy Tsukamoto Algorithm in Determining the Level of Financial Distress in Microfinance Institutions
}

\author{
Khairul $^{1}$, A. P. U. Siahaan ${ }^{1}$, Mochammad Iswan Perangin-angin ${ }^{2}$, Andre Hasudungan Lubis ${ }^{3}$, Sari Nuzullina \\ Rahmadhani ${ }^{4}$, Dahrul Siregar ${ }^{4}$ \\ ${ }^{1}$ Faculty of Science and Technology, Universitas Pembangunan Panca Budi, Medan, Indonesia \\ ${ }^{2}$ Department of Informatics, STMIK Triguna Dharma, Medan, Indonesia \\ ${ }^{3}$ Faculty of Engineering, Universitas Medan Area, Medan, Indonesia \\ ${ }^{4}$ Faculty of Economics and Business, Universitas Medan Area, Medan, Indonesia
}

\begin{abstract}
Fuzzy Tsukamoto is one method that is very flexible and tolerant of existing data. Fuzzy Tsukamoto has the advantage of being more intuitive, accepted by many, more suitable for the input received from humans rather than machines. Microfinance institutions are specialized financial institutions established to provide business development services and community empowerment, either through loans or financing in micro-scale businesses to members and the community, deposit management, and the provision of business development consulting services that are not solely for profit. The purpose of this study is to apply the fuzzy Tsukamoto method to determine the level of financial distress in microfinance institutions in the city of Medan based on the variables Liquidity Ratio, Age Firm, and Cumulative Profitability Ratio, Profitability Ratio, Financial Structure Ratio, Capital Turnover Ratio.
\end{abstract}

Keywords : Fuzzy Tsukamoto, Financial Distress, Microfinance Institutions

\section{INTRODUCTION}

Financial distress according is a condition where the company's finances are in a state of unhealthy or crisis [1]-[5] Financial distress that is quite disruptive to the company's operations is a condition that must be watched out and anticipated. Based on the explanation above, the author conducted a study of fuzzy inference systems used in determining the level of financial distress in microfinance institutions in Medan. The data used in determining the level of financial distress in microfinance institutions will be used as variables for the process of forming sets, functions of implications, the composition of rules to affirmations. Fuzzy Tsukamoto method has been widely used in solving various problems such as comparative analysis of fuzzy Tsukamoto method and Mamdani fuzzy method on comparison.

\section{FUZZY LOGIC}

Fuzzy logic is a proper way to map an input space into an output space [6]-[8]. The model of fuzzy logic is easier to understand and fuzzy logic if there is data that is not right to have tolerance. In general, fuzzy logic systems have four elements, such as:

- Base rules that contain rules sourced from experts.

- A decision-making mechanism where experts make decisions by applying the knowledge they have. 
- Fuzzification process that changes the crisp amount into fuzzy quantities.

- The defuzzification process is the opposite of the fuzzification process, which is to change the fuzzy magnitude of the result of the inference engine, to become a crisp quantity.

In system implementation, fuzzy has three parts, such as fuzzification, fuzzy inference, and defuzzification. However, the defuzzification process is optional, that is, if the conclusion has met or as expected, then there is no need to do a defuzzification process. However, if the conclusion has not been fulfilled, the defuzzification process will still be carried out. Fuzzy logic has a membership function that consists of a limit of input data values and output data values. The definition of the membership function is a graph that contains points from the limit of input data values in a membership value that is worth between 0 and 1 .

In the graph the membership function has three parts, such as the core, support, and boundary. The core part is a part of the graph which states the complete area of all fuzzy sets, then if it is expressed in a function where $\mathrm{x}$ is a member of the set $\mu(\mathrm{x})=1$. Furthermore, the second part, which is support, support or support, is a graph that states the region with membership values not 0 from the fuzzy set, then if it is expressed in a function where $\mathrm{x}$ is a member of the set $\mu(\mathrm{x})>0$. Moreover, finally, boundary or boundary. The Boundary in the membership function graph states the minimum limit value and the maximum limit of the fuzzy set, then if expressed in a function where $\mathrm{x}$ is a set member is 0 $<\mu(\mathrm{x})>1$.

Fuzzy Tsukamoto method is one method of the Fuzzy Inference System, a decision-making system [9]. In the fuzzy Tsukamoto method use rules or rules in the form of "cause-effect" or "if-then". The method of calculation from the fuzzy Tsukamoto method first is the rule formed representing the fuzzy set, then calculated the degree of membership by the rules that have been made. After obtaining the membership grade, the alpha predicate $(\alpha)$ value is sought by finding the minimum value from the value of the degree of membership. The last step, look for the output value which is the value of crisp ( $\mathrm{z}$ ) which is called the defuzzification process, which is expressed in the following equation.

$$
z=\frac{\sum_{n}^{i} \alpha(i) \cdot z(i)}{\sum_{n}^{i} z(i)}
$$

where $\alpha=$ alpha predicate (minimum value of the value of membership degrees), $\mathrm{Zi}=$ crisp value obtained from the formula of the degree of membership of the fuzzy set which is the output value, and $\mathrm{Z}=$ centered average defuzzification.

\section{MICRO FINANCIAL INSTITUTIONS}

The Financial Services Authority defines Microfinance Institutions as financial institutions specifically established to provide business development and community empowerment services, either through loans or financing in micro-scale businesses to members and the community, deposit management, and the provision of business development consulting services that are not solely seeking profit [10]-[12]. The legal basis for the establishment of Microfinance Institutions in Indonesia is:

1. Law Number 1 of 2013 concerning Microfinance Institutions (MFI Law).

2. Government Regulation Number 89 of 2014 concerning Loan Interest Rates or Yields and the Area of Business Coverage of Microfinance Institutions.

3. Regulation of the Financial Services Authority (Peraturan Otoritas Jasa Keuangan) (POJK):

a. POJK Number 12 / POJK.05 / 2014 concerning Business Licensing and Institutions of Microfinance Institutions. 
b. POJK Number 13 / POJK.05 / 2014 concerning Business Implementation of Microfinance Institutions.

c. POJK Number 14 / POJK.05 / 2014 concerning Guidance and Supervision of Microfinance Institutions.

The objectives of Microfinance Institutions are (1) to increase access to microfinance for the community, (2) to help increase economic empowerment and community productivity and (3) help increase income and welfare of the community, especially the poor and low-income groups. In achieving the goals set, the activities carried out in the form of:

1. The MFI's business activities include business development and community empowerment services, either through loans or financing in micro-scale businesses to members and the community, deposit management, and the provision of business development consulting services.

2. The business activities referred to can be carried out conventionally or based on Sharia Principles.

The types of Microfinance Institutions based on the Financial Services Authority include: Bank Desa, Lumbung Desa, Bank Pasar, Bank Pegawai, Bank Kredit Desa (BKD), Bank Kredit Kecamatan (BKK), Kredit Usaha Rakyat Kecil (KURK), Lembaga Perkreditan Kecamatan (LPK), Bank Karya Produksi (BKPD), Badan Usaha Kredit Pedesaan (BUKP), Baitul Maal wa Tamwil (BMT), Baitul Tamwil Muhammadiyah (BTM), and other similar institutions.

\section{IDENTIFICATION OF FUZZY VARIABLES}

In identifying variables, research has eight input variables and one output variable as shown in the following table.
Table 1. Research Variables

\begin{tabular}{|c|l|}
\hline Function & \multicolumn{1}{|c|}{ Variable Name } \\
\hline \multirow{5}{*}{ Input } & $\begin{array}{l}\text { T1 (Liquidity - Working Capital to } \\
\text { Total Assets) }\end{array}$ \\
\cline { 2 - 2 } & $\begin{array}{l}\text { T2 (Profitability - Retained earnings / } \\
\text { Total Assets) }\end{array}$ \\
\cline { 2 - 2 } & T3 (Profitility - ROI) \\
\cline { 2 - 2 } & $\begin{array}{l}\text { T4 (Market Value of Equity / Total } \\
\text { Liablities) }\end{array}$ \\
\cline { 2 - 2 } & T5 (Activity - Total Assets Turnover) \\
\hline Output & Micro Institution Health Level \\
\hline
\end{tabular}

Based on the previous table, the fuzzy domain can be arranged like the following table.

Table 2. Fuzzy Domain

\begin{tabular}{|c|c|c|}
\hline Variable & Fuzzy set & Range \\
\hline \multirow{3}{*}{$\begin{array}{l}\text { T1 } \\
\text { (Liquidity - } \\
\text { Working } \\
\text { Capital to } \\
\text { Total } \\
\text { Assets) }\end{array}$} & Experience financial distress & $<1$ \\
\hline & Gray area & $1<\mathrm{x}<2$ \\
\hline & $\begin{array}{l}\text { Not experiencing financial } \\
\text { distress }\end{array}$ & $>3$ \\
\hline \multirow{3}{*}{$\begin{array}{l}\text { T2 } \\
\text { (Profitabilit } \\
\text { y } \\
\text { Retained } \\
\text { earnings / } \\
\text { Total } \\
\text { Assets) } \\
\end{array}$} & Experience financial distress & $<1$ \\
\hline & Gray area & $1<\mathrm{x}<2$ \\
\hline & $\begin{array}{l}\text { Not experiencing financial } \\
\text { distress }\end{array}$ & $>3$ \\
\hline \multirow{3}{*}{$\begin{array}{l}\text { T3 } \\
\text { (Profitility } \\
\text { - ROI) }\end{array}$} & Experience financial distress & $<1$ \\
\hline & Gray area & $1<\mathrm{x}<2$ \\
\hline & $\begin{array}{l}\text { Not experiencing financial } \\
\text { distress }\end{array}$ & $>3$ \\
\hline \multirow{3}{*}{$\begin{array}{l}\text { T4 (Market } \\
\text { Value of } \\
\text { Equity / } \\
\text { Total } \\
\text { Liablities) }\end{array}$} & Experience financial distress & $<1$ \\
\hline & Gray area & $1<\mathrm{x}<2$ \\
\hline & $\begin{array}{l}\text { Not experiencing financial } \\
\text { distress }\end{array}$ & $>3$ \\
\hline \multirow{2}{*}{$\begin{array}{l}\text { T5 } \\
\text { (Activity - }\end{array}$} & Experience financial distress & $<1$ \\
\hline & Gray area & $1<\mathrm{x}<2$ \\
\hline
\end{tabular}




\begin{tabular}{|l|l|c|}
$\begin{array}{l}\text { Total Assets } \\
\text { Turnover) }\end{array}$ & $\begin{array}{l}\text { Not experiencing financial } \\
\text { distress }\end{array}$ & $>3$ \\
\hline Micro & Experience financial distress & $<1,81$ \\
\cline { 2 - 3 } $\begin{array}{l}\text { Institution } \\
\text { Health }\end{array}$ & Gray area & $1,81-$ \\
Level & $\begin{array}{l}\text { Not experiencing financial } \\
\text { distress }\end{array}$ & $>2,99$ \\
\hline
\end{tabular}

\section{RESULT AND DISCUSSION}

The data used is the data of Microfinance Institutions in Medan City in the period 2011-2016 have the following variables:

1. Liquidity Ratio

2. Age Firm and Cumulative Profitability Ratio

3. Profitability Ratio

4. Financial Structure Ratio

5. Capital Turnover Rasio

Table 3. Data on Micro Institutions Financial Reports

\begin{tabular}{|c|c|c|c|c|c|c|c|c|}
\hline $\begin{array}{l}\text { Com } \\
\text { pany }\end{array}$ & $\begin{array}{l}\mathrm{Ye} \\
\text { ar }\end{array}$ & $\begin{array}{c}W \\
\text { or } \\
k i \\
n g \\
\text { Ca } \\
\text { pit } \\
\text { al }\end{array}$ & $\begin{array}{c}\text { To } \\
\text { tal } \\
\text { As } \\
\text { set } \\
\text { s }\end{array}$ & $\begin{array}{c}\text { Re } \\
\text { tai } \\
\text { ne } \\
d \\
\text { Ea } \\
\text { rni } \\
\text { ng } \\
s\end{array}$ & $\begin{array}{l}E B \\
I T\end{array}$ & $\begin{array}{c}\text { Mar } \\
\text { ket } \\
\text { Valu } \\
\text { e of } \\
\text { Equi } \\
\text { ty }\end{array}$ & $\begin{array}{c}\text { Total } \\
\text { Liabi } \\
\text { lities }\end{array}$ & Sales \\
\hline \multirow{4}{*}{$\begin{array}{c}\text { PT } \\
\text { BPR } \\
\text { DAN } \\
\text { A } \\
\text { MA } \\
\text { NDI } \\
\text { RI }\end{array}$} & $\begin{array}{l}20 \\
13\end{array}$ & $\begin{array}{l}8.9 \\
84 . \\
30 \\
5.0 \\
0\end{array}$ & $\begin{array}{l}18 . \\
06 \\
6.0 \\
54 . \\
00\end{array}$ & $\begin{array}{l}5.0 \\
00 . \\
00 \\
0.0 \\
0\end{array}$ & $\begin{array}{l}58 \\
2.7 \\
96 . \\
00\end{array}$ & $\begin{array}{l}3.00 \\
0.00 \\
0.00\end{array}$ & $\begin{array}{l}15.7 \\
14.0 \\
53.0 \\
0\end{array}$ & $\begin{array}{l}2.13 \\
1.75 \\
0.00\end{array}$ \\
\hline & $\begin{array}{l}20 \\
14\end{array}$ & $\begin{array}{l}7.5 \\
82 . \\
78 \\
5.0 \\
0\end{array}$ & $\begin{array}{l}16 . \\
40 \\
8.4 \\
34 . \\
00\end{array}$ & $\begin{array}{l}5.0 \\
00 . \\
00 \\
0.0 \\
0\end{array}$ & $\begin{array}{l}31 \\
9.6 \\
96 . \\
00\end{array}$ & $\begin{array}{l}3.00 \\
0.00 \\
0.00\end{array}$ & $\begin{array}{l}13.7 \\
36.7 \\
37.0 \\
0\end{array}$ & $\begin{array}{l}1.91 \\
5.04 \\
2.00\end{array}$ \\
\hline & $\begin{array}{l}20 \\
15\end{array}$ & $\begin{array}{l}9.1 \\
66 . \\
25 \\
7.0 \\
0\end{array}$ & $\begin{array}{l}16 . \\
19 \\
7.6 \\
46 . \\
00\end{array}$ & $\begin{array}{l}5.0 \\
00 . \\
00 \\
0.0 \\
0\end{array}$ & $\begin{array}{l}18 \\
9.1 \\
21 . \\
00\end{array}$ & $\begin{array}{l}3.00 \\
0.00 \\
0.00\end{array}$ & $\begin{array}{l}13.3 \\
36.8 \\
22.0 \\
0\end{array}$ & $\begin{array}{l}1.76 \\
0.38 \\
4.00\end{array}$ \\
\hline & $\begin{array}{l}20 \\
16\end{array}$ & 9.8 & 17. & 5.0 & 41 & 3.00 & 14.2 & 2.12 \\
\hline
\end{tabular}

\begin{tabular}{|c|c|c|c|c|c|c|c|c|}
\hline & & $\begin{array}{l}90 . \\
78 \\
7.0 \\
0\end{array}$ & $\begin{array}{l}53 \\
2.1 \\
81 . \\
00\end{array}$ & $\begin{array}{l}00 . \\
00 \\
0.0 \\
0\end{array}$ & $\begin{array}{l}6.4 \\
69 . \\
00\end{array}$ & $\begin{array}{l}0.00 \\
0.00\end{array}$ & $\begin{array}{l}54.8 \\
89.0 \\
0\end{array}$ & $\begin{array}{l}2.23 \\
4.00\end{array}$ \\
\hline & $\begin{array}{l}20 \\
17 \\
\end{array}$ & $\begin{array}{l}8.6 \\
45 . \\
12 \\
7.0 \\
0 \\
\end{array}$ & $\begin{array}{l}15 . \\
94 \\
5.6 \\
61 . \\
00 \\
\end{array}$ & $\begin{array}{l}5.0 \\
00 . \\
00 \\
0.0 \\
0 \\
\end{array}$ & $\begin{array}{l}18 . \\
74 \\
8.0 \\
0 \\
\end{array}$ & $\begin{array}{l}3.00 \\
0.00 \\
0.00\end{array}$ & $\begin{array}{l}12.6 \\
49.6 \\
20.0 \\
0 \\
\end{array}$ & $\begin{array}{l}1.86 \\
4.56 \\
1.00 \\
\end{array}$ \\
\hline & $\begin{array}{l}20 \\
13 \\
\end{array}$ & $\begin{array}{l}16 . \\
51 \\
1.8 \\
20 . \\
00\end{array}$ & $\begin{array}{l}20 . \\
64 \\
7.0 \\
34 . \\
00 \\
\end{array}$ & $\begin{array}{l}1.6 \\
00 . \\
00 \\
0.0 \\
0\end{array}$ & $\begin{array}{l}(2 \\
52 . \\
88 \\
2.0 \\
0) \\
\end{array}$ & $\begin{array}{l}3.31 \\
3.92 \\
5.00 \\
\end{array}$ & $\begin{array}{l}17.3 \\
33.1 \\
09.0 \\
0 \\
\end{array}$ & $\begin{array}{l}4.77 \\
3.83 \\
1.00 \\
\end{array}$ \\
\hline & $\begin{array}{l}20 \\
14\end{array}$ & $\begin{array}{l}19 . \\
29 \\
9.3 \\
68 . \\
00\end{array}$ & $\begin{array}{l}30 . \\
83 \\
8.8 \\
43 . \\
00\end{array}$ & $\begin{array}{l}1.6 \\
00 . \\
00 \\
0.0 \\
0\end{array}$ & $\begin{array}{l}1.0 \\
47 . \\
11 \\
4.0 \\
0\end{array}$ & $\begin{array}{l}4.36 \\
1.03 \\
9.00\end{array}$ & $\begin{array}{l}26.4 \\
77.8 \\
04.0 \\
0\end{array}$ & $\begin{array}{l}4.68 \\
0.19 \\
2.00\end{array}$ \\
\hline $\begin{array}{c}\text { BPR } \\
\text { DUT } \\
\text { A } \\
\text { ADI } \\
\text { ART } \\
\text { A }\end{array}$ & $\begin{array}{l}20 \\
15\end{array}$ & $\begin{array}{l}19 . \\
68 \\
0.5 \\
80 . \\
00\end{array}$ & $\begin{array}{l}29 . \\
07 \\
5.6 \\
79 . \\
00\end{array}$ & $\begin{array}{l}1.6 \\
00 . \\
00 \\
0.0 \\
0\end{array}$ & $\begin{array}{l}1.2 \\
02 . \\
63 \\
1.0 \\
0\end{array}$ & $\begin{array}{l}5.56 \\
3.67 \\
0.00\end{array}$ & $\begin{array}{l}23.5 \\
12.0 \\
09.0 \\
0\end{array}$ & $\begin{array}{l}5.70 \\
4.98 \\
0.00\end{array}$ \\
\hline & $\begin{array}{l}20 \\
16 \\
\end{array}$ & $\begin{array}{l}17 . \\
99 \\
9.1 \\
98 . \\
00\end{array}$ & $\begin{array}{l}35 . \\
65 \\
8.5 \\
71 . \\
00 \\
\end{array}$ & $\begin{array}{l}1.6 \\
00 . \\
00 \\
0.0 \\
0 \\
\end{array}$ & $\begin{array}{l}1.3 \\
01 . \\
79 \\
7.0 \\
0\end{array}$ & $\begin{array}{l}6.64 \\
5.61 \\
4.00\end{array}$ & $\begin{array}{l}29.0 \\
12.9 \\
57.0 \\
0\end{array}$ & $\begin{array}{l}5.72 \\
0.79 \\
0.00\end{array}$ \\
\hline & $\begin{array}{l}20 \\
17 \\
\end{array}$ & $\begin{array}{l}16 . \\
99 \\
9.6 \\
40 . \\
00\end{array}$ & $\begin{array}{l}33 . \\
69 \\
1.9 \\
61 . \\
00 \\
\end{array}$ & $\begin{array}{l}1.6 \\
00 . \\
00 \\
0.0 \\
0 \\
\end{array}$ & $\begin{array}{l}(2 \\
0.7 \\
88 . \\
00 \\
) \\
\end{array}$ & $\begin{array}{l}8.00 \\
6.51 \\
0.00 \\
\end{array}$ & $\begin{array}{l}25.6 \\
85.4 \\
51.0 \\
0 \\
\end{array}$ & $\begin{array}{l}5.07 \\
3.50 \\
0.00 \\
\end{array}$ \\
\hline & $\begin{array}{l}20 \\
13 \\
\end{array}$ & $\begin{array}{l}31 . \\
32 \\
9.4 \\
94 . \\
00\end{array}$ & $\begin{array}{l}37 . \\
90 \\
4.7 \\
56 . \\
00\end{array}$ & $\begin{array}{l}2.0 \\
54 . \\
16 \\
5.0 \\
0\end{array}$ & $\begin{array}{l}1.5 \\
17 . \\
63 \\
4.0 \\
0\end{array}$ & $\begin{array}{l}3.55 \\
4.16 \\
5.00\end{array}$ & $\begin{array}{l}34.3 \\
50.5 \\
91.0 \\
0\end{array}$ & $\begin{array}{l}7.79 \\
0.90 \\
5.00\end{array}$ \\
\hline $\begin{array}{c}\text { BPR } \\
\text { EKA } \\
\text { PRA } \\
\text { SET } \\
\text { YA }\end{array}$ & $\begin{array}{l}20 \\
14\end{array}$ & $\begin{array}{l}28 . \\
16 \\
8.0 \\
28 . \\
00\end{array}$ & $\begin{array}{l}34 . \\
54 \\
1.1 \\
79 . \\
00\end{array}$ & $\begin{array}{l}1.3 \\
84 . \\
37 \\
8.0 \\
0\end{array}$ & $\begin{array}{l}31 \\
6.3 \\
32 . \\
00\end{array}$ & $\begin{array}{l}3.38 \\
4.37 \\
8.00\end{array}$ & $\begin{array}{l}31.1 \\
56.8 \\
01.0 \\
0\end{array}$ & $\begin{array}{l}8.25 \\
9.02 \\
8.00\end{array}$ \\
\hline & $\begin{array}{l}20 \\
15\end{array}$ & $\begin{array}{l}26 . \\
25 \\
0.0 \\
40 .\end{array}$ & $\begin{array}{l}30 . \\
61 \\
8.7 \\
70 .\end{array}$ & $\begin{array}{l}22 \\
5.5 \\
03 . \\
00\end{array}$ & $\begin{array}{l}(1 \\
08 . \\
87 \\
6.0\end{array}$ & $\begin{array}{l}2.22 \\
5.50 \\
3.00\end{array}$ & $\begin{array}{l}28.3 \\
93.2 \\
67.0 \\
0\end{array}$ & $\begin{array}{l}7.22 \\
9.32 \\
6.00\end{array}$ \\
\hline
\end{tabular}




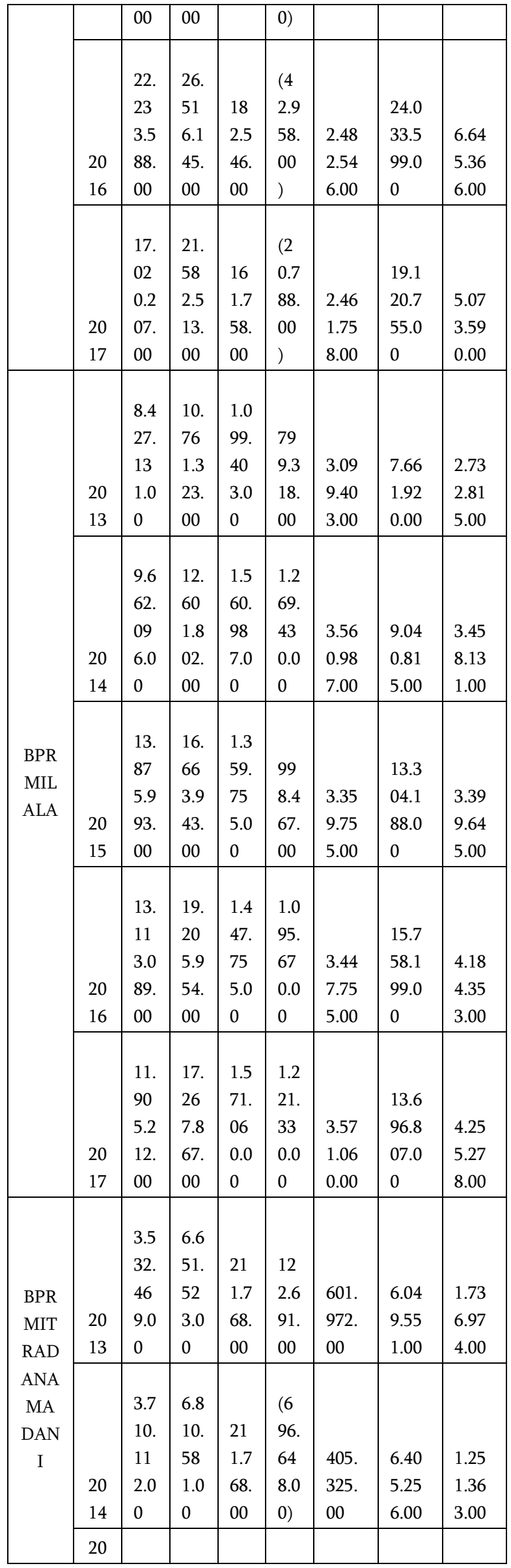

\begin{tabular}{|c|c|c|c|c|c|c|c|c|}
\hline & 15 & $\begin{array}{l}4.6 \\
30 . \\
48 \\
6.0 \\
0 \\
\end{array}$ & $\begin{array}{l}6.0 \\
83 . \\
92 \\
8.0 \\
0 \\
\end{array}$ & $\begin{array}{l}21 \\
1.7 \\
68 . \\
00\end{array}$ & $\begin{array}{l}5 \\
08 . \\
77 \\
0.0 \\
0)\end{array}$ & $\begin{array}{l}346 . \\
554 . \\
00\end{array}$ & $\begin{array}{l}5.73 \\
7.37 \\
4.00\end{array}$ & $\begin{array}{l}1.01 \\
8.88 \\
2.00\end{array}$ \\
\hline & $\begin{array}{l}20 \\
16 \\
\end{array}$ & $\begin{array}{l}5.8 \\
75 . \\
10 \\
1.0 \\
0 \\
\end{array}$ & $\begin{array}{l}8.2 \\
04 . \\
44 \\
8.0 \\
0 \\
\end{array}$ & $\begin{array}{l}21 \\
1.7 \\
68 . \\
00 \\
\end{array}$ & $\begin{array}{l}16 \\
3.4 \\
72 . \\
00 \\
\end{array}$ & $\begin{array}{l}758 . \\
390 . \\
00\end{array}$ & $\begin{array}{l}7.44 \\
6.05 \\
8.00 \\
\end{array}$ & $\begin{array}{l}1.46 \\
8.19 \\
1.00 \\
\end{array}$ \\
\hline & $\begin{array}{l}20 \\
17\end{array}$ & $\begin{array}{l}7.4 \\
41 . \\
15 \\
0.0 \\
0\end{array}$ & $\begin{array}{l}10 . \\
95 \\
5.9 \\
47 . \\
00\end{array}$ & $\begin{array}{l}21 \\
1.7 \\
68 . \\
00\end{array}$ & $\begin{array}{l}24 \\
6.1 \\
89 . \\
00\end{array}$ & $\begin{array}{l}1.00 \\
2.27 \\
8.00\end{array}$ & $\begin{array}{l}9.95 \\
3.66 \\
9.00\end{array}$ & $\begin{array}{l}2.19 \\
9.12 \\
4.00\end{array}$ \\
\hline & $\begin{array}{l}20 \\
13\end{array}$ & $\begin{array}{l}5.1 \\
16 . \\
57 \\
7.0 \\
0\end{array}$ & $\begin{array}{l}13 . \\
31 \\
0.8 \\
40 . \\
00\end{array}$ & $\begin{array}{l}1.4 \\
89 . \\
14 \\
1.0 \\
0\end{array}$ & $\begin{array}{l}(3 \\
56 . \\
10 \\
8.0 \\
0)\end{array}$ & $\begin{array}{l}510 . \\
859 . \\
00\end{array}$ & $\begin{array}{l}12.7 \\
99.9 \\
81.0 \\
0\end{array}$ & $\begin{array}{l}1.74 \\
6.36 \\
7.00\end{array}$ \\
\hline & $\begin{array}{l}20 \\
14\end{array}$ & $\begin{array}{l}4.8 \\
51 . \\
42 \\
1.0 \\
0\end{array}$ & $\begin{array}{l}10 . \\
43 \\
7.7 \\
64 . \\
00\end{array}$ & $\begin{array}{l}1.0 \\
73 . \\
78 \\
3.0 \\
0\end{array}$ & $\begin{array}{l}42 \\
6.5 \\
90 . \\
00\end{array}$ & $\begin{array}{l}926 . \\
217 . \\
00\end{array}$ & $\begin{array}{l}9.51 \\
1.54 \\
7.00\end{array}$ & $\begin{array}{l}1.83 \\
8.45 \\
6.00\end{array}$ \\
\hline $\begin{array}{c}\text { BPR } \\
\text { TAT } \\
\text { A } \\
\text { PAT } \\
\text { UMB } \\
\text { AK }\end{array}$ & $\begin{array}{l}20 \\
15 \\
\end{array}$ & $\begin{array}{l}5.5 \\
41 . \\
87 \\
3.0 \\
0 \\
\end{array}$ & $\begin{array}{l}14 . \\
03 \\
0.4 \\
77 . \\
00 \\
\end{array}$ & $\begin{array}{l}1.2 \\
09 . \\
44 \\
8.0 \\
0 \\
\end{array}$ & $\begin{array}{l}(8 \\
2.3 \\
41 . \\
00 \\
) \\
\end{array}$ & $\begin{array}{l}790 . \\
552 . \\
00\end{array}$ & $\begin{array}{l}13.2 \\
39.9 \\
25.0 \\
0\end{array}$ & $\begin{array}{l}2.00 \\
3.11 \\
4.00 \\
\end{array}$ \\
\hline & $\begin{array}{l}20 \\
16 \\
\end{array}$ & $\begin{array}{l}6.5 \\
32 . \\
50 \\
7.0 \\
0 \\
\end{array}$ & $\begin{array}{l}14 . \\
85 \\
9.0 \\
39 . \\
00 \\
\end{array}$ & $\begin{array}{l}1.0 \\
21 . \\
52 \\
0.0 \\
0 \\
\end{array}$ & $\begin{array}{l}20 \\
5.7 \\
35 . \\
00 \\
\end{array}$ & $\begin{array}{l}978 . \\
480 . \\
00\end{array}$ & $\begin{array}{l}13.8 \\
80.5 \\
59.0 \\
0 \\
\end{array}$ & $\begin{array}{l}2.41 \\
9.85 \\
2.00 \\
\end{array}$ \\
\hline & $\begin{array}{l}20 \\
17 \\
\end{array}$ & $\begin{array}{l}7.5 \\
24 . \\
55 \\
0.0 \\
0 \\
\end{array}$ & $\begin{array}{l}15 . \\
04 \\
6.5 \\
33 . \\
00 \\
\end{array}$ & $\begin{array}{l}93 \\
7.2 \\
58 . \\
00 \\
\end{array}$ & $\begin{array}{l}13 \\
6.4 \\
90 . \\
00\end{array}$ & $\begin{array}{l}1.06 \\
2.74 \\
2.00 \\
\end{array}$ & $\begin{array}{l}13.9 \\
83.7 \\
91.0 \\
0\end{array}$ & $\begin{array}{l}2.62 \\
1.21 \\
1.00\end{array}$ \\
\hline $\begin{array}{c}\text { BPR } \\
\text { WA } \\
\text { HAN } \\
\text { A } \\
\text { BER } \\
\text { SAM }\end{array}$ & $\begin{array}{l}20 \\
13\end{array}$ & $\begin{array}{l}8.0 \\
19 . \\
37 \\
0.0 \\
0\end{array}$ & $\begin{array}{l}9.8 \\
46 . \\
69 \\
0.0 \\
0\end{array}$ & $\begin{array}{l}85 \\
4.3 \\
83 . \\
00\end{array}$ & $\begin{array}{l}(2 \\
8.8 \\
41 . \\
00 \\
)\end{array}$ & $\begin{array}{l}1.14 \\
5.61 \\
7.00\end{array}$ & $\begin{array}{l}8.70 \\
1.07 \\
3.00\end{array}$ & $\begin{array}{l}1.76 \\
6.08 \\
0.00\end{array}$ \\
\hline $\begin{array}{c}\mathrm{A} \\
\mathrm{KPU} \\
\mathrm{M}\end{array}$ & $\begin{array}{l}20 \\
14\end{array}$ & $\begin{array}{l}9.4 \\
11 . \\
02\end{array}$ & $\begin{array}{l}11 . \\
87 \\
5.5\end{array}$ & $\begin{array}{l}92 \\
1.7 \\
15 .\end{array}$ & $\begin{array}{l}(5 \\
4.8 \\
36 . \\
\end{array}$ & $\begin{array}{l}1.07 \\
8.28 \\
5.00\end{array}$ & $\begin{array}{l}10.7 \\
97.2 \\
70.0\end{array}$ & $\begin{array}{l}2.33 \\
9.91 \\
7.00\end{array}$ \\
\hline
\end{tabular}




\begin{tabular}{|l|l|l|l|l|l|l|l|l|}
\hline & & 3.0 & 55. & 00 & 00 & & 0 & \\
& 0 & 00 & & ) & & & \\
\cline { 3 - 9 } & & 9.3 & 12. & 1.6 & $(6$ & & & \\
& 26. & 54 & 09. & 62. & & 11.1 & \\
& 95 & 0.7 & 22 & 59 & 1.39 & 50.0 & 2.63 \\
& 20 & 5.0 & 98. & 8.0 & 1.0 & 0.77 & 26.0 & 1.88 \\
15 & 0 & 00 & 0 & $0)$ & 2.00 & 0 & 7.00 \\
\hline & & & & & & & \\
& & 11. & 16. & 1.4 & & & & \\
& 27 & 06 & 87. & 15 & & 14.5 & \\
& 4.2 & 8.6 & 14 & 4.7 & 1.51 & 55.8 & 3.08 \\
& 09. & 71. & 0.0 & 66. & 2.86 & 11.0 & 7.07 \\
& 16 & 00 & 00 & 0 & 00 & 0.00 & 0 & 6.00 \\
\hline & & & & & & & \\
& 13. & 17. & 1.2 & & & & \\
& 18 & 96 & 09. & 29 & & 16.1 & \\
& & 0.5 & 0.9 & 24 & 7.5 & 1.79 & 70.1 & 3.44 \\
& 73. & 15. & 5.0 & 93. & 0.75 & 60.0 & 6.11 \\
& 00 & 00 & 0 & 00 & 5.00 & 0 & 1.00 \\
\hline
\end{tabular}

Based on the data in the previous table, the Z-Score value is obtained as follows:

Table 4. Z-Score value

\begin{tabular}{|c|l|}
\hline$Z$-Score & \multicolumn{1}{|c|}{ Information } \\
\hline$<1,81$ & Experience financial distress \\
\hline $1,81-2,99$ & Gray area \\
\hline$>2,99$ & Not experiencing financial distress \\
\hline
\end{tabular}

Table 5. Calculation Results of Financial Distress

\begin{tabular}{|c|c|c|c|}
\hline No & Company & Z-Score & Result \\
\hline \multirow{5}{*}{1} & \multirow{5}{*}{$\begin{array}{c}\text { PT BPR } \\
\text { DANA } \\
\text { MANDIRI }\end{array}$} & 1.267760301 & $\begin{array}{l}\text { Experience Financial } \\
\text { Distress }\end{array}$ \\
\hline & & 1.232143962 & $\begin{array}{l}\text { Experience Financial } \\
\text { Distress }\end{array}$ \\
\hline & & 1.331572596 & $\begin{array}{l}\text { Experience Financial } \\
\text { Distress }\end{array}$ \\
\hline & & 1.344797639 & $\begin{array}{l}\text { Experience Financial } \\
\text { Distress }\end{array}$ \\
\hline & & 1.289863945 & $\begin{array}{l}\text { Experience Financial } \\
\text { Distress }\end{array}$ \\
\hline \multirow{2}{*}{2} & \multirow{2}{*}{$\begin{array}{l}\text { BPR DUTA } \\
\text { ADIARTA }\end{array}$} & 1.357930639 & $\begin{array}{l}\text { Experience Financial } \\
\text { Distress }\end{array}$ \\
\hline & & 1.229400138 & $\begin{array}{l}\text { Experience Financial } \\
\text { Distress }\end{array}$ \\
\hline
\end{tabular}

\begin{tabular}{|c|c|c|c|}
\hline & & 1.352772445 & $\begin{array}{l}\text { Experience Financial } \\
\text { Distress }\end{array}$ \\
\hline & & 1.077741983 & $\begin{array}{l}\text { Experience Financial } \\
\text { Distress }\end{array}$ \\
\hline & & 0.997886231 & $\begin{array}{l}\text { Experience Financial } \\
\text { Distress }\end{array}$ \\
\hline \multirow{5}{*}{3} & \multirow{5}{*}{$\begin{array}{c}\text { BPR EKA } \\
\text { PRASETYA }\end{array}$} & 1.456409326 & $\begin{array}{l}\text { Experience Financial } \\
\text { Distress }\end{array}$ \\
\hline & & 1.395401984 & $\begin{array}{l}\text { Experience Financial } \\
\text { Distress }\end{array}$ \\
\hline & & 1.308786148 & $\begin{array}{l}\text { Experience Financial } \\
\text { Distress }\end{array}$ \\
\hline & & 1.32144805 & $\begin{array}{l}\text { Experience Financial } \\
\text { Distress }\end{array}$ \\
\hline & & 1.264240943 & $\begin{array}{l}\text { Experience Financial } \\
\text { Distress }\end{array}$ \\
\hline \multirow{5}{*}{4} & \multirow{5}{*}{ BPR MILALA } & 1.803828139 & $\begin{array}{l}\text { Experience Financial } \\
\text { Distress }\end{array}$ \\
\hline & & 2.209063382 & GreyArea \\
\hline & & 1.650210142 & $\begin{array}{l}\text { Experience Financial } \\
\text { Distress }\end{array}$ \\
\hline & & 1.44695486 & $\begin{array}{l}\text { Experience Financial } \\
\text { Distress }\end{array}$ \\
\hline & & 1.572528132 & $\begin{array}{l}\text { Experience Financial } \\
\text { Distress }\end{array}$ \\
\hline \multirow{5}{*}{5} & \multirow{5}{*}{$\begin{array}{c}\text { BPR } \\
\text { MITRADAN } \\
\text { A MADANI }\end{array}$} & 1.056949685 & $\begin{array}{l}\text { Experience Financial } \\
\text { Distress }\end{array}$ \\
\hline & & 0.567605706 & $\begin{array}{l}\text { Experience Financial } \\
\text { Distress }\end{array}$ \\
\hline & & 0.882673009 & $\begin{array}{l}\text { Experience Financial } \\
\text { Distress }\end{array}$ \\
\hline & & 1.195912628 & $\begin{array}{l}\text { Experience Financial } \\
\text { Distress }\end{array}$ \\
\hline & & 1.173314475 & $\begin{array}{l}\text { Experience Financial } \\
\text { Distress }\end{array}$ \\
\hline \multirow{5}{*}{6} & \multirow{5}{*}{$\begin{array}{c}\text { BPR TATA } \\
\text { PATUMBAK }\end{array}$} & 0.662247803 & $\begin{array}{l}\text { Experience Financial } \\
\text { Distress }\end{array}$ \\
\hline & & 1.092749503 & $\begin{array}{l}\text { Experience Financial } \\
\text { Distress }\end{array}$ \\
\hline & & 1.235532577 & $\begin{array}{l}\text { Experience Financial } \\
\text { Distress }\end{array}$ \\
\hline & & 1.363928132 & $\begin{array}{l}\text { Experience Financial } \\
\text { Distress }\end{array}$ \\
\hline & & 0.9244177 & $\begin{array}{l}\text { Experience Financial } \\
\text { Distress }\end{array}$ \\
\hline \multirow{2}{*}{7} & \multirow{2}{*}{$\begin{array}{c}\text { BPR } \\
\text { WAHANA } \\
\text { BERSAMA } \\
\text { KPUM }\end{array}$} & 1.329940746 & $\begin{array}{l}\text { Experience Financial } \\
\text { Distress }\end{array}$ \\
\hline & & 1.313060103 & $\begin{array}{l}\text { Experience Financial } \\
\text { Distress }\end{array}$ \\
\hline
\end{tabular}




\begin{tabular}{|l|l|l|}
\hline 1.156598838 & $\begin{array}{l}\text { Experience Financial } \\
\text { Distress }\end{array}$ \\
\cline { 2 - 3 } & 1.239081633 & $\begin{array}{l}\text { Experience Financial } \\
\text { Distress }\end{array}$ \\
\cline { 2 - 3 } & 1.27420825 & $\begin{array}{l}\text { Experience Financial } \\
\text { Distress }\end{array}$ \\
\hline
\end{tabular}

\section{CONCLUSION}

Based on the results and discussion, some conclusions can be drawn. Fuzzy inference system provides a reasonably good process quality value for assessing the level of health of microfinance institutions in Medan. It can be seen in the MSE value generated by the fuzzy inference system almost close to 0 , that is BPR MILAL IX. The results of the calculation of fuzzy inference systems are quite useful to be used in assessing the level of health of the company; this can be seen in the value of the level of accuracy of MAPE which is quite small.

\section{REFERENCES}

[1] A. Kyereboah-Coleman, "The impact of capital structure on the performance of microfinance institutions," J. Risk Financ., vol. 8, no. 1, pp. 56-71, Jan. 2007.

[2] A. K. Sari, H. Saputra, and A. P. U. P. U. Siahaan, "Financial Distress Analysis on Indonesia Stock Exchange Companies," Int. J. Innov. Res. Multidiscip. F., vol. 4, no. 3, pp. 73-74, 2018.

[3] H. A. Hasibuan, R. B. Purba, and A. P. U. Siahaan, "Productivity Assessment (Performance, Motivation, and Job Training) using Profile Matching," Int. J. Econ. Manag. Stud., vol. 3, no. 6, pp. 73-77, 2016.

[4] H. M. Ritonga, H. A. Hasibuan, and A. P. U. Siahaan, "Credit Assessment in Determining The Feasibility of Debtors Using Profile Matching," Int. J. Bus. Manag. Invent., vol. 6, no. 1, pp. 73-79, 2017.
[5] H. M. Ritonga, A. P. U. Siahaan, and Suginam, "Marketing Strategy through Markov Optimization to Predict Sales on Specific Periods," Int. J. Innov. Res. Multidiscip. F., vol. 3, no. 8, pp. 184-190, 2017.

[6] K. S. S, W. A. Kusuma, and A. Buono, "Fuzzybased Spectral Alignment for Correcting DNA Sequence from Next Generation Sequencer," TELKOMNIKA (Telecommunication Comput. Electron. Control., vol. 14, no. 2, p. 707, Jun. 2016.

[7] A. P. U. Siahaan, "Determination of Thesis Preceptor and Examiner Based on Specification of Teaching Using Fuzzy Logic."

[8] R. F. Wijaya, Y. M. Tondang, and A. P. U. Siahaan, "Take Off and Landing Prediction using Fuzzy Logic," Int. J. Recent Trends Eng. Res., vol. 2, no. 12, pp. 127-134, 2016.

[9] W. Chang, C. Ku, and W. Chang, "Fuzzy control with passivity synthesis for continuous affine Takagi-Sugeno fuzzy systems," Int. J. Intell. Comput. Cybern., vol. 2, no. 2, pp. 386408, Jun. 2009.

[10] A. Kyereboah-Coleman and K. A. Osei, "Outreach and profitability of microfinance institutions: the role of governance," J. Econ. Stud., vol. 35, no. 3, pp. 236-248, Aug. 2008.

[11] D. Bhanot and V. Bapat, "Sustainability index of micro finance institutions (MFIs) and contributory factors," Int. J. Soc. Econ., vol. 42, no. 4, pp. 387-403, Apr. 2015.

[12] A. K. Sari, H. Saputra, and A. P. U. Siahaan, "Effect of Fiscal Independence and Local Revenue Against Human Development Index," Int. J. Bus. Manag. Invent., vol. 6, no. 7, pp. 62-65, 2017. 